\title{
Differentially-Keyed IR-UWB Signals for MA with Differential-Detection Receiver
}

\author{
Walid Mahmoud \\ Communications and Networks Engineering Department, Prince Sultan University, Riyadh, KSA \\ Email:wmahmoud@gmail.com
}

Received 27 April 2014; revised 25 May 2014; accepted 17 June 2014

Copyright ( 2014 by author and Scientific Research Publishing Inc. This work is licensed under the Creative Commons Attribution International License (CC BY). http://creativecommons.org/licenses/by/4.0/ (c) (i) 0 pen Access

\begin{abstract}
Noncoherent communication receivers (differential-detectors) have simple design, however, they always incur bit error rate $(B E R)$ performance loss up to $3 \mathrm{~dB}$ compared to coherent receivers. In this paper, a differential-detector is proposed for impulse radio ultra wideband (IR-UWB) communication systems. The system employs bit-level differential phase shift keying (DPSK) combined with code division (CD) for IR-UWB signals to support multiple-access (MA). It is analyzed under additive white Gaussian noise (AWGN) corrupted by multiple-access interference (MAI) channel. Its BER performance is compared against a reference coherent receiver using MonteCarlo simulation method. A closed form expression for its average probability of error is derived analytically. Simulation results and theoretical analysis confirm the applicability of the proposed differential-detector for IR-UWB communication systems.
\end{abstract}

\section{Keywords}

Impulse-Radio Ultra-Wideband, Code Division Multiple Access, Multiple Access Interference, Bit Error Rate, Differential-Detection

\section{Introduction}

The federal communications commission (FCC) in USA has approved UWB technology for commercial use in February 2002 [1]. UWB signal is characterized by having a transmission bandwidth greater than $500 \mathrm{MHz}$ or a signal whose bandwidth to the center frequency ratio is greater than twenty percents. The bandwidth is defined as the difference between $-10 \mathrm{~dB}$ cut-off frequencies from the spectral peak-power value of UWB signal [2]. UWB has been accepted as an alternative physical layer for wireless personal area networks (WPANs) applications [3]. UWB communication systems depending on their propagated radio are classified as either impulseradio ultra-wideband (IR-UWB) or multi-carrier ultra-wideband (MC-UWB) [4] [5]. 
Transmitted signal in IR-UWB communication systems consists of a series of short-duration pulses of nanosecond order or less transmitted at baseband [6]. Thereby the energy of the signal is spread over a broad frequency range in the available UWB spectrum from $(3.1$ - 10.6) GHz, with very low power spectral density. Because of this, UWB technology can coexist with other radio services, achieve high throughput, and share the spectrum for multiple-access (MA). Although IR-UWB is intended to work at baseband level, some practical systems still utilize a carrier signal [7]-[9].

In this paper, we present a noncoherent communication system that employs bit-level DPSK modulation combined with code division (CD) on the transmitted IR-UWB signals to support multiple-access (MA). The system is analyzed under the additive white Gaussian noise (AWGN) corrupted by multiple-access interference (MAI) channel. Multiple access capability is investigated and expressed in mathematical equation as a function of active users in the system. Our proposed receiver implements differential-detection on received signal to detect the originally transmitted information bit, which is referred to as DS-DPSK. Its BER performance is compared with the BER of a reference coherent receiver reported in [10], which is referred to as DS-PAM because it employs antipodal modulation on transmitted IR-UWB signals. Comparison results indicated that the performance of our system is slightly worse than that of DS-PAM system; however, our system has simple structure that results in less design costs and power consumption.

This paper is organized as follows. Mathematical model for the proposed system is provided in Section 2. Theoretical analysis and error probability derivation are provided in Section 3. Simulation results are presented in Section 4. Finally, the conclusion is drawn in Section 5.

\section{Proposed DS-DPSK System Model}

\subsection{Transmitter}

The transmitter in DS-DPSK system is similar to that in [10], except that the system use bit-level differential encoding and the PAM Modulator is replaced by DPSK Modulator as shown in Figure 1.

The transmit signal for the $k^{\text {th }}$ user is given by

$$
S_{t r}^{(k)}=\sum_{j=-\infty}^{\infty} \sum_{n=0}^{N_{c}-1} d_{j}^{(k)} C_{n}^{(k)} p_{t r}\left(t-j T_{b}-n T_{c}\right),
$$

where $d_{j}^{(k)}=d_{j-1}^{(k)} b_{j}^{(k)}$ is the $j^{\text {th }}$ differentially encoded bit for all $b_{j}^{(k)}=2 \times b_{\left[\frac{j}{N_{s}}\right.}^{(k)}-1 \in\{-1,1\}, \quad N_{s}$ is the pulse repetition time, $c_{n}^{k}$ is the used gold code sequence, $p_{t r}(t)$ is the transmitted 2nd derivative Gaussian pulse, $T_{c}$ is the pulse repetition period, $N_{c}$ is the number of pulses per bit and the bit period is $T_{b}=N_{c} \times T_{c}$.

\subsection{Channel}

The channel is the additive white Gaussian noise (AWGN) corrupted by multiple-access interference (MAI) channel that has two sources of noise. The first source is the receiver noise, denoted as $n(t)$ and represents the additive random thermal noise at receiver input. This noise is modeled as a white Gaussian random process that has zero-mean and variance $\sigma_{n}^{2}$, i.e., $N\left(0, \sigma_{n}^{2}\right)$, with two-sided power spectral density equal to $\frac{N_{o}}{2}$. The second source is the MAI that represents the aggregate effect of having more than one active user in the system simultaneously. The MAI is approximated as a white Gaussian random process on the basis of central limit theorem.

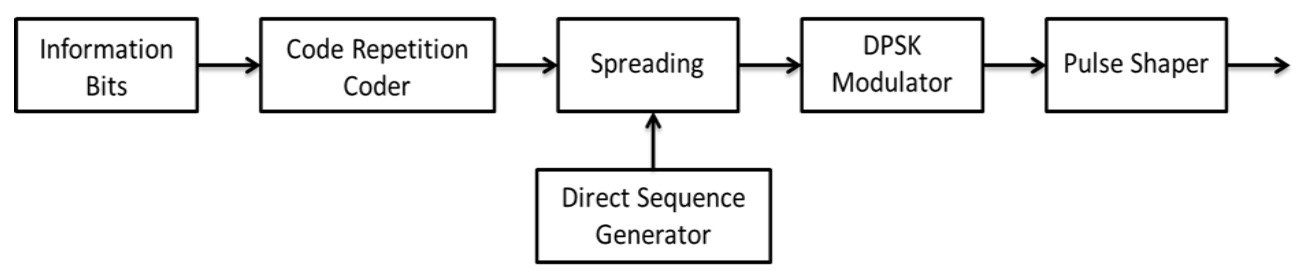




\subsection{Receiver}

When there are $N_{u}$ active users in the system, the received signal is given by

$$
r(t)=\sum_{k=1}^{N_{u}} S_{\text {rec }}^{(k)}(t)+n(t),
$$

where $S_{\text {rec }}^{(k)}(t)$ is the received signal of $k^{t h}$ user at receiver input, and $n(t)$ is the added receiver noise.

Bit-level differential encoding at transmitter side allows for bit-level differential detection at receiver side. In this scheme, the previous signaling interval (i.e., bit-period long) is used to recover the originally transmitted data bit from the current signaling interval. Therefore, receiver device should have two branches, namely branch A and branch B, to represent $r(t)$ and $r\left(t-T_{b}\right)$ as shown in Figure 2.

At receiver input each user is recognized by a unique Pseudo-Noise (PN) code. The PN Code Generator in Figure 2 exists mainly to generate the unique code for the user currently receiving data. The variable statistics at each branch, namely $\alpha_{a}$ and $\alpha_{b}$, are the result of correlating $r(t)$ and $r\left(t-T_{b}\right)$ with the desired-user PN code over bit-period. Multiplying $\alpha_{a}$ and $\alpha_{b}$ is equivalent to extracting the sign or the phase difference between the current and previous signaling intervals. The decision variable, $Z$, at the mixer-output is then compared against the threshold to recover the originally transmitted information bit.

\section{Receiver Analysis}

\subsection{Theoretical Analysis}

In this subsection the variable statistics like $\alpha_{a}, \alpha_{b}$, and the decision variable $Z$ shown in Figure 2 are analyzed, and expressed mathematically. The expression for the average probability of error in the proposed receiver is derived using the obtained mathematical expressions.

\subsubsection{Branch A}

Referring to Figure 2, correlator output is given by

$$
\alpha_{a}=\int_{0}^{T_{b}} r(t) v(t) \mathrm{d} t .
$$

The assumption hereafter is that user 1 will be the desired user, and we are going to detect the $l^{\text {th }}$ data bit. In this case, the template can be defined as

$$
v(t)=\sum_{m=0}^{N_{c}-1} c_{m}^{(1)} p_{\text {rec }}\left(t-l T_{b}-m T_{c}\right),
$$

and the received signal after simplification is given by

$$
r(t)=\sum_{n=0}^{N_{c}-1} d_{l}^{(1)} c_{n}^{(1)} p_{\text {rec }}\left(t-l T_{b}-n T_{c}\right)+n_{s},
$$

where $n_{s}=\sum_{k=2}^{N_{u}} S_{\text {rec }}^{(k)}(t)+n(t)$ is the noise source including MAI and receiver noise $n(t)$, and excluding the signal of the desired user. $S_{\text {rec }}^{(k)}(t)$ is the received signal of $k^{\text {th }}$ user at receiver input. Referring to (3), $\alpha_{a}$ in the $I^{\text {th }}$ bit-period is given by

$$
\alpha_{a}=\int_{0}^{T_{b}}\left\{\sum_{n=0}^{N_{c}-1} d_{l}^{(1)} c_{n}^{(1)} p_{\text {rec }}\left(t-n T_{c}\right)+n_{s}\right\} \times \sum_{m=0}^{N_{c}-1} c_{m}^{(1)} p_{\text {rec }}\left(t-m T_{c}\right) \mathrm{d} t .
$$

Let $\alpha_{a}=m_{a}+n_{a}$, where $m_{a}$ and $n_{a}$ are the desired signal and noise components at Branch A, respectively.

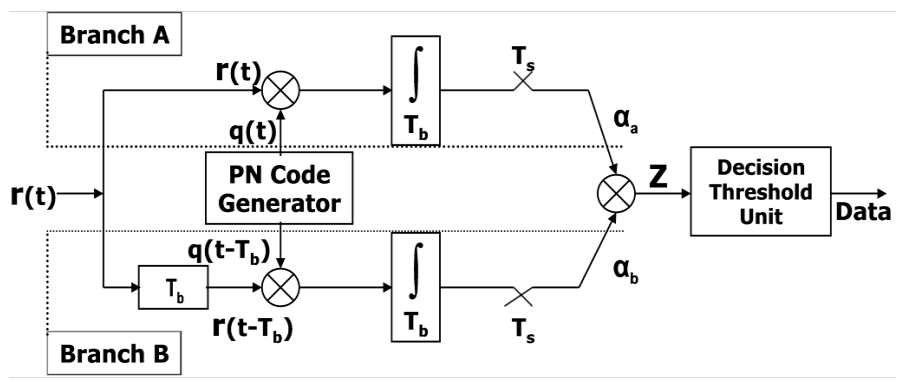

Figure 2. Proposed differential-detection MA receiver (DS-DPSK). 
The desired signal component $m_{a}$ is given by

$$
m_{a}=\int_{0}^{T_{b}}\left\{\sum_{n=0}^{N_{c}-1} d_{l}^{(1)} c_{n}^{(1)} p_{\text {rec }}\left(t-n T_{c}\right)\right\} \times \sum_{m=0}^{N_{c}-1} c_{m}^{(1)} p_{\text {rec }}\left(t-m T_{c}\right) \mathrm{d} t=N_{c} E_{p} d_{l}^{(1)}=E_{b} d_{l}^{(1)},
$$

and the noise component $n_{a}$ is given by

$$
n_{a}=\int_{0}^{T_{b}} n_{s} \times \sum_{m=0}^{N_{c}-1} c_{m}^{(1)} p_{\text {rec }}\left(t-m T_{c}\right) \mathrm{d} t .
$$

Therefore $n_{a}$ can be expressed as

$$
n_{a}=\int_{0}^{T_{b}}\left\{\sum_{k=2}^{N_{u}} S_{r e c}^{(k)}(t)+n(t)\right\} \times \sum_{m=0}^{N_{c}-1} c_{m}^{(1)} p_{\text {rec }}\left(t-m T_{c}\right) \mathrm{d} t .
$$

Furthermore, $n_{a}$ can be simplified and expressed as $n_{a}=n_{a m}+n_{a r}$, where $n_{a m}$ and $n_{a r}$ are the noise components at Branch $A$ due to MAI and receiver noise, respectively. The noise component due to MAI, $n_{a m}$, is given by

$$
n_{a m}=\int_{0}^{T_{b}} \sum_{k=2}^{N_{u}} S_{r e c}^{(k)}(t) \times \sum_{m=0}^{N_{c}-1} c_{m}^{(1)} p_{\text {rec }}\left(t-m T_{c}\right) \mathrm{d} t .
$$

After simplifying (10) above, $n_{a m}$ can be expressed as

$$
n_{a m}=\sum_{k=2}^{N_{u}} d_{l}^{(k)}\left\{c_{k, 1}^{(0)}\right\},
$$

where $\left\{c_{k, 1}^{(0)}\right\}$ is the synchronous cross-correlation function between the desired and interfering users during the $l^{\text {th }}$ bit-period. Now the expression for the noise component due to receiver noise, $n_{a r}$, is given by

$$
n_{a r}=\int_{0}^{T_{b}} n(t) \times \sum_{m=0}^{N_{c}-1} c_{m}^{(1)} p_{r e c}\left(t-m T_{c}\right) \mathrm{d} t .
$$

Under the assumption in [11], $n_{a r}$ has Gaussian distribution with zero-mean and variance $\sigma_{\text {reca }}^{2}$ that is given by

$$
\sigma_{\text {reca }}^{2}=N_{c} E_{p} \sigma_{n}^{2}=E_{b} \sigma_{n}^{2} \text {. }
$$

Thus, the variable statistic $\alpha_{a}$ for Branch $A$ is given by

$$
\alpha_{a}=m_{a}+n_{a r}+n_{a m} .
$$

The obtained variable statistic will assist in deriving the final expression for the average probability of error in the proposed receiver.

\subsubsection{Branch B}

Due to similarity of derivations with Branch A, except for the bit-period delay that should be considered, only necessary derivations relevant to Branch B are included here. By following similar steps, the variable statistic $\alpha_{b}$ is given by

$$
\alpha_{b}=m_{b}+n_{b r}+n_{b m},
$$

where the right-hand side terms are the desired signal, receiver noise and MAI components in Branch B, respectively. The desired signal component $m_{b}$ is given by

$$
m_{b}=\int_{0}^{T_{b}} \sum_{n=0}^{N_{c}-1} d_{l}^{(1)} c_{n}^{(1)} p_{\text {rec }}\left(t-T_{b}-n T_{c}\right) \times \sum_{m=0}^{N_{c}-1} c_{m}^{(1)} p_{\text {rec }}\left(t-T_{b}-m T_{c}\right) \mathrm{d} t=N_{c} E_{p} d_{l-1}^{(1)}=E_{b} d_{l-1}^{(1)} .
$$

The noise component due to receiver noise, $n_{b r}$, is given by

$$
n_{b r}=\int_{0}^{T_{b}} n\left(t-T_{b}\right) \times \sum_{m=0}^{N_{c}-1} c_{m}^{(1)} p_{\text {rec }}\left(t-T_{b}-m T_{c}\right) \mathrm{d} t .
$$

Similar to $n_{a r}$ in (12), $n_{b r}$ has Gaussian distribution with zero-mean and variance $\sigma_{\text {recb }}^{2}$ that is given by

$$
\sigma_{\text {recb }}^{2}=N_{c} E_{p} \sigma_{n}^{2}=E_{b} \sigma_{n}^{2} \text {. }
$$

The noise component due to MAI, $n_{b m}$, is given by

$$
n_{b m}=\sum_{k=2}^{N_{u}} d_{l-1}^{(k)}\left\{C_{k, 1}^{(0)}\right\},
$$


where $\left\{C_{k, 1}^{(0)}\right\}$ is the cross-correlation function between the desired and interfering users during the $(l-1)$-th bit-period.

\subsection{Error Probability Derivation}

The randomness of the used PN code sequences with the existence of more than one active-user concurrently in the system permits the approximation of MAI terms, $n_{a m}$ in (11) and $n_{b m}$ in (19) as Gaussian random processes [12]. Therefore, the variable statistic $\alpha_{a}$ can be expressed as

$$
\alpha_{a}=m_{a}+n_{a},
$$

where $n_{a}=n_{a r}+n_{a m}$, and the variable statistic $\alpha_{b}$ can be expressed as

$$
\alpha_{b}=m_{b}+n_{b}
$$

where $n_{b}=n_{b r}+n_{b m}$. Referring to Figure 2, the decision variable $Z$ is equal to the multiplication of the variable statistics $\alpha_{a}$ and $\alpha_{b}$. Assuming that the data bit 1 is transmitted, then, the current and previous bits of DPSK signal are the same, hence $m_{a}=m_{b}$. Therefore, $Z$ after simplification can be expressed as

$$
Z=m_{a}^{2}+m_{a b}+n_{a b},
$$

where $m_{a b}=m_{a} \times\left[n_{a}+n_{b}\right]$ and $n_{a b}=n_{a} \times n_{b}$. If the desired signal component $m_{a}$ in (7) is squared, then we have

$$
m_{a}^{2}=\left(N_{c} E_{p} d_{l}^{(1)}\right)^{2}=\left(E_{b} d_{l}^{(1)}\right)^{2}=E_{b}^{2} .
$$

Note that receiver noise $n(t)$ is modeled as AWGN process with zero-mean (DC power) and variance $\sigma_{n}^{2}$ (AC power) that is equal to the spectral (total) power of this process. Therefore, $\sigma_{n}^{2}$ is equal to $\left(\frac{N_{o}}{2}\right)$. Substituting the value of $\sigma_{n}^{2}$ in (13), the expected value for $n_{a r}$ is given by $E\left[n_{a r}\right]=0$, and its variance $\operatorname{var}\left[n_{a r}\right]=E_{b} \sigma_{n}^{2}=E_{b}\left(\frac{N_{o}}{2}\right)$. The expected value for $n_{a m}$ is given by $E\left[n_{a m}\right]=0$ and its variance $\operatorname{var}\left[n_{a m}\right]=(K-1) \times\left(\frac{E_{b}}{N_{c}}\right)^{2}$. After approximating MAI term with a Gaussian random process, and considering the effect of other active users in the system, the statistical value for the total noise at Branch A, $n_{a}$, is given by

$$
\begin{gathered}
E\left[n_{a}\right]=0, \\
\operatorname{var}\left[n_{a}\right]=E_{b}\left(\frac{N_{o}}{2}\right)+(K-1) \times\left(\frac{E_{b}}{N_{c}}\right)^{2} .
\end{gathered}
$$

Since $n_{b}$ and $n_{a}$ expected to have similar statistical values due to randomness and independence of Gaussian processes, the new process is the sum of their statistics. Therefore, $m_{a b}$ has zero-mean expected value and variance given by

$$
\operatorname{var}\left[m_{a b}\right]=E_{b} N_{o}+2 \times(K-1) \times\left(\frac{E_{b}}{N_{c}}\right)^{2} .
$$

Statistical values for $n_{a b}$ term in (22) are derived using Shannon's sampling theorem [13]. Thus, the variable $n_{a b}$ can be expressed as

$$
n_{a b}=\int_{0}^{T_{b}} n_{a} \times n_{b} \mathrm{~d} t=\int_{0}^{T_{b}} g t \times g\left(t+T_{b}\right) \mathrm{d} t,
$$

where $g(t)$ is a Gaussian random process with zero-mean and variance $\sigma_{g}^{2}=\frac{N_{o}}{2}+\frac{1}{N_{c}^{2}}$, which has autocorrelation function that is given by 


$$
R(\tau) \stackrel{\text { def }}{=} E[g(t)+g(t+\tau)]=\left(\frac{N_{o}}{2}+\frac{1}{N_{c}^{2}}\right) \delta(t-\tau) .
$$

The sampled version of $n_{a b}$ can be expressed as

$$
n_{a b}=\sum_{x=0}^{N} g(x) \delta\left(t-\frac{x}{2 \beta}\right) \sum_{y=0}^{N} g(y+N) \delta\left(t-\frac{y}{2 \beta}\right),
$$

where $N=2 \beta T_{b}$ is the number of independent samples per bit, and $\beta$ is the bandwidth of the 2nd derivative Gaussian pulse. Both $g(x)$ and $g(y+N)$ are independent and identically distributed (i.i.d) samples from the sampled version of the Gaussian process. Each sample has zero-mean and variance equal to $\frac{N_{o}}{(2 \sqrt{c l})}+\frac{1}{\left(\sqrt{c l} N_{c}^{2}\right)}$ as a result of energy normalization, where $\mathrm{cl}$ is the length of the used PN code sequence. Because the bit-period $\left(T_{b}\right)$ is much greater than the sampling-period $\left(\frac{1}{2 \beta}\right)$, we use the following approximation

$$
\int_{0}^{T_{b}} \delta\left(t-\frac{x}{2 \beta}\right) \delta\left(t-\frac{y}{2 \beta}\right) \mathrm{d} t= \begin{cases}1: & x=y \\ 0: & x \neq y,\end{cases}
$$

thus $n_{a b}$ can be further simplified and is given by

$$
n_{a b}=\sum_{x=0}^{N} g(x) g(x+N)=\sum_{x=0}^{N} N_{x},
$$

when $y=x$. Therefore, the statistics for $N_{x}$ is given by $E\left[N_{x}\right]=0$, and

$$
\operatorname{var}\left[N_{x}\right]=\operatorname{var}[g(x)] \times \operatorname{var}[g(x+N)]=\left[\frac{N_{o}}{(2 \sqrt{c l})}+\frac{1}{\left(\sqrt{c l} N_{c}^{2}\right)}\right]^{2}=\frac{1}{c l\left[\frac{N_{o}}{2}+\frac{1}{N_{c}^{2}}\right]^{2}} .
$$

Because each sample has Gaussian distribution, the sum of the entire $N$ independent samples also has Gaussian distribution based on central limit theorem. Therefore, $n_{a b}$ has Gaussian distribution with zero-mean expected value and variance given by

$$
\operatorname{var}\left[n_{a b}\right]=\frac{(k-1) 2 \beta T_{b}}{c l} \times\left[\frac{N_{o}}{2}+\frac{1}{N_{c}^{2}}\right]^{2},
$$

after including the effect of other active users in the system. The signal-to-noise ratio $\left(\gamma_{b}\right)$ is given by

$$
\gamma_{b}=\frac{m_{a}^{2}}{\operatorname{var}\left[m_{a b}\right]+\operatorname{var}\left[n_{a b}\right]} .
$$

Substitute Equations (23), (25) and (31) in (32), the analytical expression for $\gamma_{b}$ is given by

$$
\gamma_{b}=\frac{E_{b}^{2}}{E_{b} N_{o}+2 \times(K-1) \times\left(\frac{E_{b}}{N_{c}}\right)^{2}+\frac{2 \beta T_{b}}{c l} \times\left[\frac{N_{o}}{2}+\frac{1}{N_{c}^{2}}\right]^{2}} .
$$

Substitute $\gamma_{b}$ in the known expression for the average probability of error using DPSK receiver in AWGN channel we have

$$
P_{-} e=0.5 \exp \left(-\gamma_{b}\right),
$$

which is the analytical average probability of error in the proposed receiver.

\section{Simulation Results}

Simulation results for DS-DPSK (proposed) and DS-PAM (reference) systems using Matlab are presented in 
this section. Simulation parameters in both systems are identical in order to have fair and unbiased comparisons. The used 2nd derivative Gaussian pulse is given by

$$
W_{\frac{t x}{r x}}(t)=\left[1-4 p\left(\frac{t}{\tau_{m}}\right)^{2}\right] \exp \left[-2 p\left(\frac{t}{\tau_{m}}\right)^{2}\right],
$$

with pulse width $\tau_{m}=0.2 \mathrm{~ns}$ and pulse period $T_{w}=0.5 \mathrm{~ns}$. The length of used gold sequences $\mathrm{cl}=7$ and pulse repetition period $T_{c}=1 \mathrm{~ns}$. Thus, the expected data rate for the proposed system is $R_{b}=\frac{1}{c l \times T_{c}} \approx 150 \mathrm{Mbps}$ per user. In both systems the interferers are 1, 2 and 3 such that the total number of active users in the system is 4 users.

\subsection{Proposed vs Reference}

Performance comparison between DS-DPSK and DS-PAM receivers for the required SNR vs BER for different number of active users in the system is shown in Figure 3.

Because any communications receiver capable of achieving BER performance level $10^{-3}$ is considered functional receiver, $S N R$ values for different number of active users at this level in both receivers are used as the
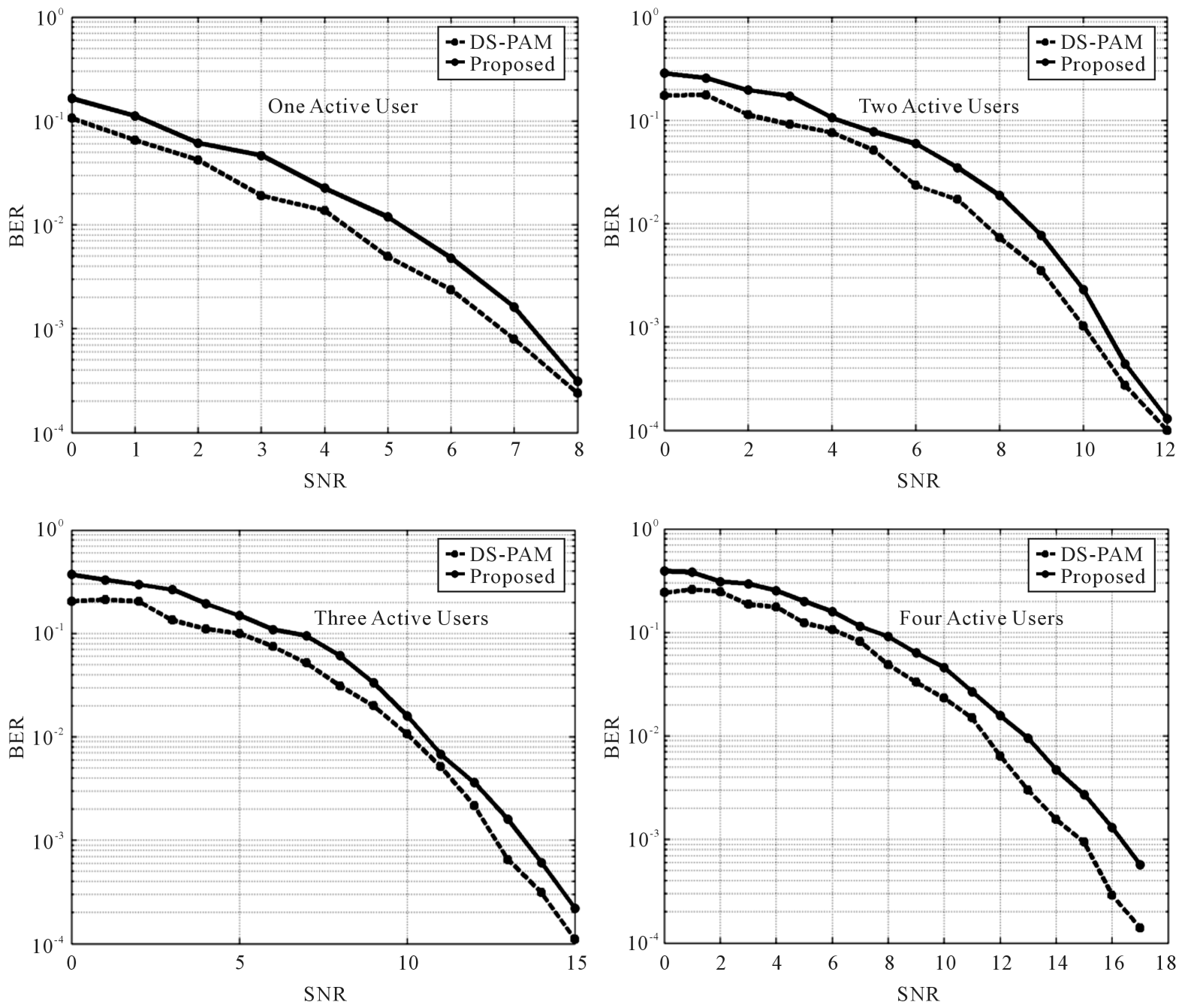

Figure 3. BER vs SNR (DS-PAM \& DS-DPSK receivers). 
comparison base. Such SNR values are extracted from Figure 3 and tabulated in Table 1 along with the difference and percentage difference (\%PD) values.

From Figure 3, BER performance for same SNR values of proposed system is slightly worse than that of DS-PAM system. It is known in the literature that coherent (DS-PAM) receivers are superior in terms of BER performance compared to noncoherent (DS-DPSK) receivers as a result of demodulation process. In such receivers, the carrier used for demodulation at receiver side is in phase and frequency synchronism with the carrier used for modulation at transmitter side. This requires some sophisticated circuitry such as the phase-locked loop circuit, which is not a requirement for noncoherent receivers. Thus, coherent receivers are rendered complex from design perspective, costly and consume extra power. However, noncohenret receivers are known to be simple in their design, less costly and consume less power with BER performance degradation up to $3 \mathrm{~dB}$ compared to coherent receivers.

From Table 1, for the case of four active users in both systems, it is shown that the maximum increase in SNR required to achieve $10^{-3}$ BER using DS-DPSK receiver is $1.2 \mathrm{~dB}$. Since this value is less than $3 \mathrm{~dB}$-the expected increase in $S N R$ as a result of using noncoherent receivers such as differential detectors, this renders our proposed receiver a practical IR-UWB differential-detector.

The slight performance improvement in the proposed system is the result of using gold code sequences in conjunction with DPSK modulation. Gold code sequences have noise-like behavior responsible in general for improving multiple-access capability of communication systems, and in particular, in improving spectral properties of the transmitted DPSK signal. In addition, working principle of DS-DPSK receiver in using template signal that experienced the same channel effects is another factor in improving its performance.

\subsection{Efficiency of DS-DPSK}

SNR versus BER graphs for the case of having 1, 2, 3 and 4 active users in the proposed system are shown in Figure 4. The behavior of these graphs has motivated the definition of a new performance metric, which is referred to as multiple-access efficiency (MAE), which is intended to test the efficiency of DS-DPSK as multipleaccess receiver.

Referring to Figure 4, one can see convergence in distance among the graphs in the figure and such convergence is more among the graphs representing higher number of active users in the system. MAE metric is the mathematical expression that represents the inverse of this convergence in percentage. It reflects the required increase in $S N R$ values as a result of gradual increase in the number of active users in the system. MAE metric is given by

$$
\mathrm{MAE}=\left[10^{\left(\frac{S N R_{c c}-S N R_{p c}}{10}\right)}\right]^{-1} \times 100 \%,
$$

where $S N R_{c c}$ and $S N R_{p c}$ are defined as follows. By drawing a horizontal line at $B E R=10^{-3}$ in Figure 4, if the movement is from the intersection point on the single-user graph to the intersection point on the two-users

\begin{tabular}{ccccc} 
Table 1. Performance comparison DS-PAM \& DS-DPSK. \\
\hline Receivers/Users & \multicolumn{5}{c}{ Number of Active Users } \\
\cline { 2 - 5 } & One & Two & Three & Four \\
\hline DS-PAM $(a)$ & 6.8 & 10 & 12.6 & 15 \\
DS-DPSK & 7.2 & 10.6 & 13.7 & 16.2 \\
Difference $(b)$ & 0.4 & 0.6 & 1.1 & 1.2 \\
$\%$ PD $^{\mathrm{a}}=\frac{(b)}{(a)} \times 100$ & 5.8 & 6 & 8.7 & 8 \\
\hline
\end{tabular}

${ }^{\mathrm{a}}$ Percentage difference. 


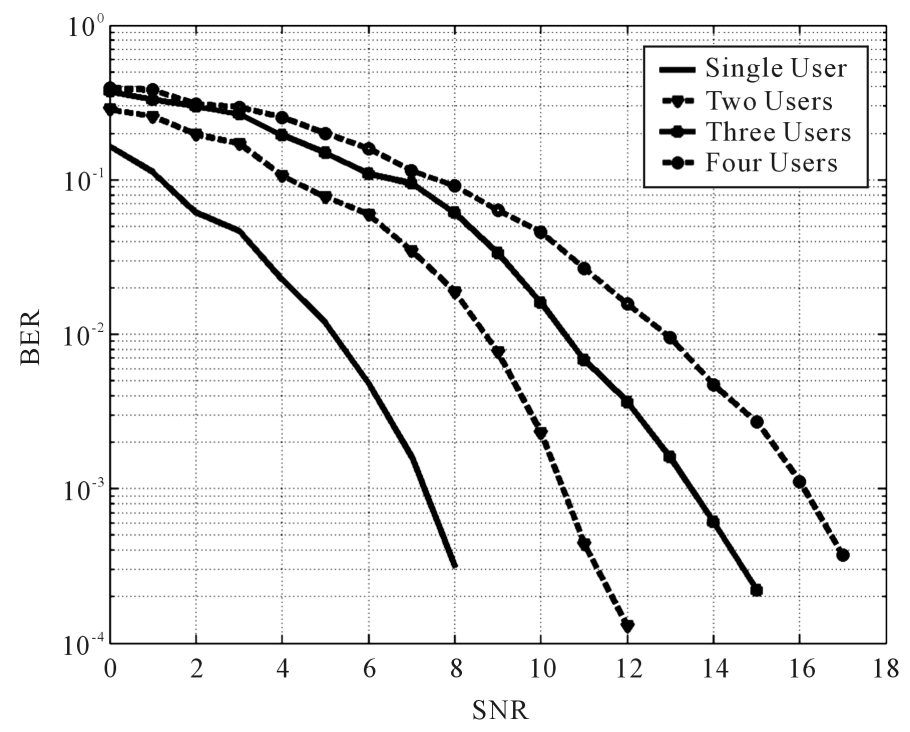

Figure 4. BER vs SNR (MAE of DS-DPSK receiver).

graph, then $S N R_{p c}$ is the value of the first point and $S N R_{c c}$ is the value of the second point. Proceeding in this way while moving from graph 2 to graph 3, and from graph 3 to graph 4, respectively, the calculated MAE values are $45.7 \%$, $49 \%$, and $56.5 \%$.

The slight gradual increase in SNR values above indicates that DS-DPSK receiver is capable of handling the increase in the number of active users in the system as long as MAE not reached $100 \%$ value. This performance metric has confirmed the feasibility of the proposed system to support multiple-access. MAE metric can be used to define the upper bound for the required increase in SNR, especially when it approaches $100 \%$ value. At this value, the maximum number of active users that DS-DPSK system can support is also approached.

\subsection{Analysis vs Simulation Results}

Comparison between analytical and simulation results for different number of active users in the proposed system is shown in Figure 5.

From Figure 5, the obtained simulation results are in good match with the derived analytical results represented by the mathematical expressions describing DS-DPSK system.

In other words, both graphs for each case of having difference number of active users are close to each other and change in tandem. Thus, the comparison confirmed the correctness of the followed analytical methods and the derived mathematical expressions presented herein. The small gap between graphs of one-active user in the system is the result of approximating MAI as Gaussian random process. Based on central limit theorem, this approximation becomes more valid as the number of active users in the system increases, which is evident on the graphs for more active users in the system.

\section{Conclusions}

In this paper a noncoherent IR-UWB differential-detector (DS-DPSK) that supports multiple-access was proposed. The proposed system was analyzed under AWGN corrupted by multiple-access interference channel. Closed-form expression for the average probability of error in the proposed system was derived analytically. It is compared against a reference coherent receiver (DS-PAM) for BER performance for the cases of having 1, 2, 3 and 4 active users in the system using Matlab based on Monte-Carlo simulation method. Its capability to support multiple users was investigated and new multiple-access efficiency metric was defined. Simulation results were in good match with the derived analytical expressions describing our proposed system. Despite its slight BER performance loss as a noncohenret receiver relative to DS-PAM coherent receiver, its performance relative to other differential-detectors and its simple structure, which yields less design costs and power consumption, are the major advantages. Thus, it is a suitable IR-UWB communication system that supports multiple-access. 



Figure 5. Analysis vs simulation results (DS-DPSK receiver).

Given the fact that mathematical analysis and simulation results confirmed the applicability of our proposed system, a possible future work is to devise other mechanisms to further improve its BER performance.

\section{Acknowledgements}

The author would like to warmly thank peer reviewers for their help in reviewing this manuscript.

\section{References}

[1] FCC Notice of Proposed Rulemaking (2002) Revision of Part 15 of the Commission’s Rules Regarding Ultra-Wideband Transmission Systems. Federal Communications Commission, Washington DC, 98-135.

[2] Nekkogar, F. (2006) Ultra-Wideband Communications: Fundamentals and Applications. Prentice Hall, Upper Saddle River.

[3] IEEE P802.15-02/105r25, SG3a Alternate PHY Selection Criteria.

[4] Reed, J. (2005) An Introduction to Ultra Wideband Communications Systems. Prentice Hall, New Jersey.

[5] Woo, C., August, N. and Dong, S. (2005) Signaling and Multiple Access Techniques for Ultra Wideband 4G Wireless Communication Systems. IEEE Wireless Communications Journal, 12, 46-55.

[6] Win, M. and Scholtz, R. (1998) Impulse Radio: How It Works. IEEE Communications Letters, 2, 36-38. http://dx.doi.org/10.1109/4234.660796

[7] Fisher, R., et al. (2004) DS-UWB Physical Layer Submission to 802.15 Task Group 3a. IEEE 802.15-04/137r3, July 2004. 
[8] Wentzloff, D., Blazquez, R., Lee, F., Ginsburg, B., Powell, J. and Chandrakasan, A. (2005) System Design Considerations for Ultra-Wideband Communication. IEEE Journal on Communications, 43, 114-121.

[9] Runkle, P., McCorkle, J., Miller, T. and Welborn, M. (2003) DS-CDMA: The Modulation Technology of Choice for UWB Communications. IEEE Conference on Ultra Wideband Systems and Technologies, Virginia, 16-19 November 2003, 364-368. http://dx.doi.org/10.1109/UWBST.2003.1267865

[10] Bai, Z. and Kwak, K. (2005) Analysis of Multiuser DS-PAM and TH-PPM UWB Systems in Data and Image Transmission. Proceedings of IEEE International Conference on Wireless Communications, Networking and Mobile Computing, Vol. 1, Incheon, 23-26 September 2005, 324-327.

[11] Scholtz, A. (1993) Multiple Access with Time-Hopping Impulse Modulation. Proceedings of IEEE Conference on Military Communications, Boston, 11-14 October 1993, 11-14. http://dx.doi.org/10.1109/MILCOM.1993.408628

[12] Idriss, A., Moorfeld, R., Zeisberg, S. and Finger, A. (2005) Performance of Coherent and Non-Coherent Receivers of UWB Communication. Second IFIP International Conference on Wireless and Optical Communication Networks, Germany, 6-8 March 2005, 117-122.

[13] Marco, P. and Gerard, J. (2004) Analysis and Comparison of Autocorrelation Receivers for IR-UWB Signals Based on Differential Detection. IEEE International Conference on Acoustics, Speech, and Signal Processing, Vol. 4, Netherlands, May 2004, 513-516. 
Scientific Research Publishing (SCIRP) is one of the largest Open Access journal publishers. It is currently publishing more than 200 open access, online, peer-reviewed journals covering a wide range of academic disciplines. SCIRP serves the worldwide academic communities and contributes to the progress and application of science with its publication.

Other selected journals from SCIRP are listed as below. Submit your manuscript to us via either submit@scirp.org or Online Submission Portal.
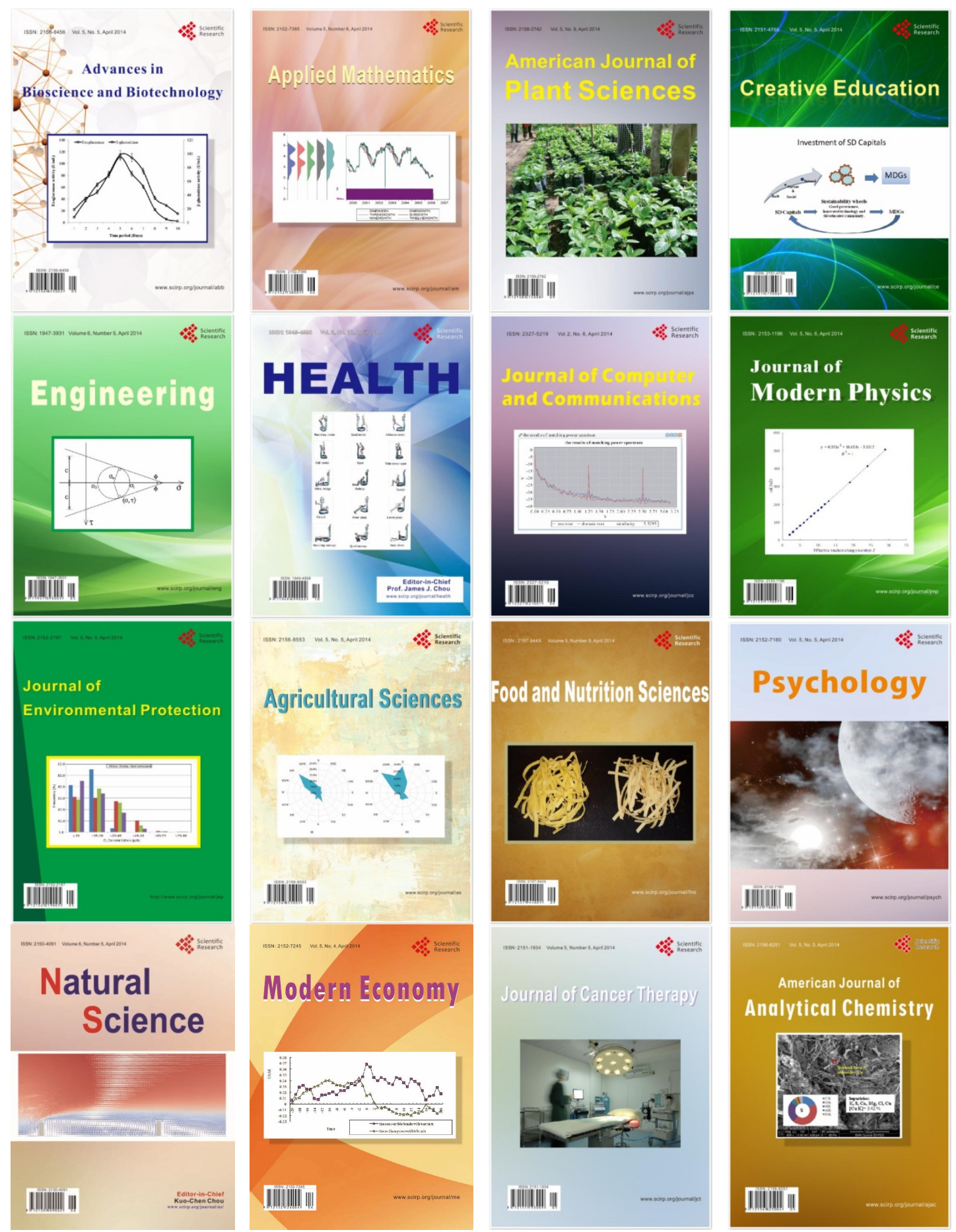